\title{
14 nights of intermittent hypoxia elevate daytime blood pressure and sympathetic activity in healthy humans
}

\author{
R. Tamisier*,\#, J.L. Pépin*,\#, J. Rémy\#, J.P. Baguet", J.A. Taylor ${ }^{+}$, \\ J.W. Weiss ${ }^{\S}$ and P. Lévy*\#
}

ABSTRACT: Obstructive sleep apnoea syndrome (OSAS) causes nocturnal chronic intermittent hypoxia (IH) that contributes to excess cardiovascular morbidity. To explore the consequences of $\mathrm{IH}$, we used our recently developed model of nocturnal IH in healthy humans to characterise the profile of this blood pressure increase, to determine if it is sustained and to explore potential physiological mechanisms.

We performed 24-h ambulatory monitoring of blood pressure in 12 healthy subjects before and after 2 weeks of IH exposure. We also assessed systemic haemodynamics, muscle sympathetic nerve activity (MSNA), ischaemic calf blood flow responses and baroreflex gain. We obtained blood samples for inflammatory markers before, during and after exposure. IH significantly increased daytime ambulatory blood pressure after a single night of exposure $(3 \mathrm{mmHg}$ for mean and diastolic) and further increased daytime pressures after 2 weeks of exposure $(8 \mathrm{mmHg}$ systolic and $5 \mathrm{mmHg}$ diastolic). Mean \pm SD MSNA increased across the exposure (17.2 \pm 5.1 versus $21.7 \pm 7.3$ bursts $\left.\mathrm{min}^{-1} ; \mathrm{p}<0.01\right)$ and baroreflex control of sympathetic outflow declined from $-965.3 \pm 375.1$ to $-598.4 \pm 162.6 \mathrm{AlU} \cdot \mathrm{min}^{-1} \cdot \mathrm{mmHg}^{-1}(p<0.01)$. There were no evident changes in either vascular reactivity or systemic inflammatory markers.

These data are the first to show that the arterial pressure rise is sustained throughout the waking hours beyond the acute phase immediately after exposure. Moreover, they may suggest that sympathoactivation induced by IH likely contributes to blood pressure elevation and may derive from reduced baroreflex inhibition. These mechanisms may reflect those underlying the blood pressure elevation associated with OSAS.

KEYWORDS: Atherosclerosis, hypertension, pathophysiology, sleep apnoea

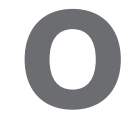

bstructive sleep apnoea syndrome (OSAS) is highly prevalent in western countries, with an age-related prevalence ranging $5-15 \%$ up to $60 \mathrm{yrs}$ of age [1]. The primary health concern for OSAS is increased cardiovascular morbidity. OSAS is independently associated with hypertension [2], and confers an increased risk for fatal and nonfatal cardiovascular complications [3-5]. Hence, OSAS is a significant health concern in Western countries $[6,7]$.

Considering these epidemiologic associations, the pathophysiological link between OSAS and cardiovascular disease must be defined to consider potential avenues for treatment. Several mechanisms have been proposed that could link OSAS to cardiovascular disease: the high vascular sympathetic tone exhibited by OSAS patients may result in elevated systemic resistance and, hence, elevated pressure [8-11]; impaired arterial vasodilatory capacity [12-14] may contribute to elevation of blood pressure and lead to vascular disease; and sustained inflammation may lead to endothelial damage [15] and contribute to atherosclerosis. Although this is not an exhaustive list, we proposed to focus on these in the present study. A primary stimulus for these alterations in OSAS is nocturnal exposure to chronic intermittent hypoxia $(\mathrm{CIH})$. Animal models of $\mathrm{CIH}$ alone [16] or with the other stimuli that characterise OSAS (i.e. respiratory effort, asphyxia and arousal from sleep) [17] show elevated blood pressure during the non-CIH portion of the day. Data derived from the former model suggest that the blood pressure elevation results from sympathetic activation [18]. This may require an intact chemoreflex loop $[19,20]$, but data also suggests that following $\mathrm{CIH}$, arterial baroreflex gain is decreased [21, 22].

\section{AFFILIATIONS}

*Sleep Laboratory and EFCR, Dept of Rehabilitation and Physiology, *HP2 Laboratory, INSERM U1042, EA 3745 Joseph Fourier University, 'Dept of Cardiology and Hypertension, University Hospital, Grenoble, France. ${ }^{+}$Dept of Physical Medicine and Rehabilitation, Harvard Medical School,

${ }^{\S}$ Pulmonary and Sleep Research Laboratory, Division of Pulmonary, Critical Care and Sleep Medicine, Beth Israel Deaconess Medical Center, Boston, MA, USA.

CORRESPONDENCE

R. Tamisier

Laboratoire d'Exploration Fonctionnelle Respiratoire Pôle Rééducation et Physiologie Centre Hospitalier Universitaire de Grenoble BP 217

38043 Grenoble Cedex 9

France

E-mail: rtamisier@chu-grenoble.fr

Received:

Dec 292009

Accepted after revision:

May 152010

First published online:

June 042010 
Although animal models have improved our understanding, there are specific aspects of human physiology that may not be adequately represented. Clinical research has been crucial to define the relationships between sleep apnoea and cardiovascular morbidity $[23,24]$. However, confounders such as obesity, age, and metabolic disorders prevent clear understanding of the pathophysiologic effects of nightly exposure to $\mathrm{CIH}$. Therefore, to explore whether the blood pressure rise is sustained beyond the acute phase immediately after intermittent hypoxia (IH) exposure, we used our recently developed model of intermittent hypoxia in healthy humans that induces unstable ventilation and sleep fragmentation similar to that observed in OSAS patients [25]. Interestingly, after 7 and 14 days of $\mathrm{CIH}$, our model induced an increase in both acute isocapnic hypoxic ventilatory response and acute hyperoxic hypercapnic ventilatory response [25]. This was also reported for isocapnic hypoxic ventilatory response after 4 days of waking IH [26]. Moreover, using the same model, FOSTER et al. [27] demonstrated changes in cardio- and cerebrovascular responses to acute hypoxia following exposure to intermittent hypoxia.

Moreover, we explored three possible contributors to the IHinduced elevation in blood pressure and atherogenesis: greater sympathetic activation, lesser post-occlusive-mediated vasorelaxation and systemic inflammation. We hypothesised that after 2 weeks of exposure to IH, arterial blood pressure would remain elevated and this would relate to increased sympathetic activity to muscle vasculature, decreased flow-mediated dilation and increased circulating biomarkers of inflammation.

\section{METHODS}

\section{Subjects}

12 healthy, nonsmoking, normotensive subjects (two of whom were female), with a mean \pm SD age of $23 \pm 6$ yrs (body mass index (BMI) $21.7 \pm 1.9 \mathrm{~kg} \cdot \mathrm{m}^{-2}$ ), who were free of vasoactive medications, completed the study. A screening history and physical exam was performed to assure that each subject was free of cardiac, pulmonary or neurologic diseases. Individuals who had travelled to or lived at an altitude $>2,500 \mathrm{~m}$ in the 6 months prior to the study were excluded. All females were studied during the first week following menses and tested negative for pregnancy before exposure, after one night of $\mathrm{CIH}$ and at the end of the protocol. This report encompasses data not previously reported, but acquired from subjects that completed the 2-week protocol described previously [25].

The sample size of 12 subjects was based on the expected changes in sympathetic activity and diastolic blood pressure measured by 24-h ambulatory blood pressure measurement. The study was powered at $80 \%$ to observe a $20 \%$ increase in sympathetic activity from a baseline level of $15.6 \pm 5.6$ bursts. $\mathrm{min}^{-1}[28]$ and a $10-\mathrm{mmHg}(11 \%)$ increase in daytime diastolic blood pressure from a baseline of $82.8 \pm 9.1 \mathrm{mmHg}$ [29] with an $\alpha$ of 0.05 .

All subjects provided written informed consent approved by the ethical committee at the Grenoble University Hospital Center (Grenoble, France).

\section{IH exposure}

This CIH exposure has been previously reported [25].
Following a two-night adaptation to the environment (room air) and a one-night adaptation to an intermediate $\mathrm{IH}$ level, subjects were exposed to $8 \mathrm{~h}$ severe IH between the 23:00 and 07:00 $\mathrm{h}$ for 14 consecutive nights. The IH stimulus was intermittent poikilocapnic hypoxia, i.e. inspiratory oxygen fraction $\left(\mathrm{FI}, \mathrm{O}_{2}\right)$ was controlled and carbon dioxide was allowed to fluctuate normally. For all nights, subjects slept with a nasal cannula in a commercially available hypoxia tent (Hypoxico Inc., New York, $\mathrm{NY}$, USA). The tent exposed subjects to an $\mathrm{FI}, \mathrm{O}_{2}$ of 0.15 for the intermediate $\mathrm{IH}$ level and 0.13 for the 14 nights of severe $\mathrm{IH}$. The tent was continuously flushed and the oxygen fraction in the tent was continuously monitored (Maxtec OM-25 MEI; Maxtec Inc., Salt Lake City, UT, USA) to limit rebreathing. The nasal cannula restored oxygen saturation via a 15-s bolus of oxygen every $120 \mathrm{~s}$. Oxygen saturation was monitored continuously (BlueNight; SleepInnov Technology, Moirans, France) and oxygen boluses were adjusted between 1.5 and $2 \mathrm{~L} \cdot \mathrm{min}^{-1}$ to achieve an $85-95 \%$ range of oxygen desaturationresaturation. The combination of tent and nasal cannula allowed for 30 oxygen desaturation-resaturation sequences per hour. This level and frequency of desaturation is clinically analogous to severe OSAS (fig. 1a and b).

\section{General procedures}

Diurnal blood pressure patterns as well as urine and blood samples were obtained before exposure, after one and 13 nights (i.e. 2 weeks) of severe $\mathrm{CIH}$, and after 5 days of recovery. Cardiovascular measurements were performed before exposure and following the 14th night (2 weeks) of severe IH (fig. 1c).

Ambulatory blood pressure was measured in the dominant arm over $24 \mathrm{~h}$ at 15-min intervals (ABP monitor 90207; Spacelabs Healthcare, Issaquah, WA, USA). Blood pressure acquisition began at 09:00 $\mathrm{h}$ and ended $24 \mathrm{~h}$ later. Measurements before exposure and after recovery represent room air conditions for the entire $24 \mathrm{~h}$, whereas measurements after one and 13 nights of IH represent daytime room air and nighttime IH conditions.

Cardiovascular measurements were recorded between 08:00 and 12:00 h after subjects had fasted overnight and with subjects in the supine position during room air breathing. Testing was performed in the following order, with $>15 \mathrm{~min}$ separating each: resting supine measurements, modified Oxford baroreflex test, reactive hyperaemia assessment and hypoxic challenge. All data were digitalised continuously at $500 \mathrm{~Hz}$ to a computer and analysed subsequently with signal processing software (Windaq; Dataq Instruments, Akron, OH, USA).

Echocardiographic assessment was performed in the afternoon of the same day at the same time for all subjects.

\section{Measurements}

Heart period (i.e. heart rate and $\mathrm{R}-\mathrm{R}$ interval) was obtained from a three-lead electrocardiogram. Arterial pressures were measured in the right arm at 1-min intervals via an automated arm-cuff sphygmomanometer (Dinamap; Critikon, Tampa, FL, USA) and on a beat-by-beat basis via digital photoplethysmography (Finapres ${ }^{\circledR}$; Ohmeda, Louisville, CO, USA). We obtained peroneal nerve recordings via standard sympathetic microneurographic procedures with tungsten microelectrodes, 

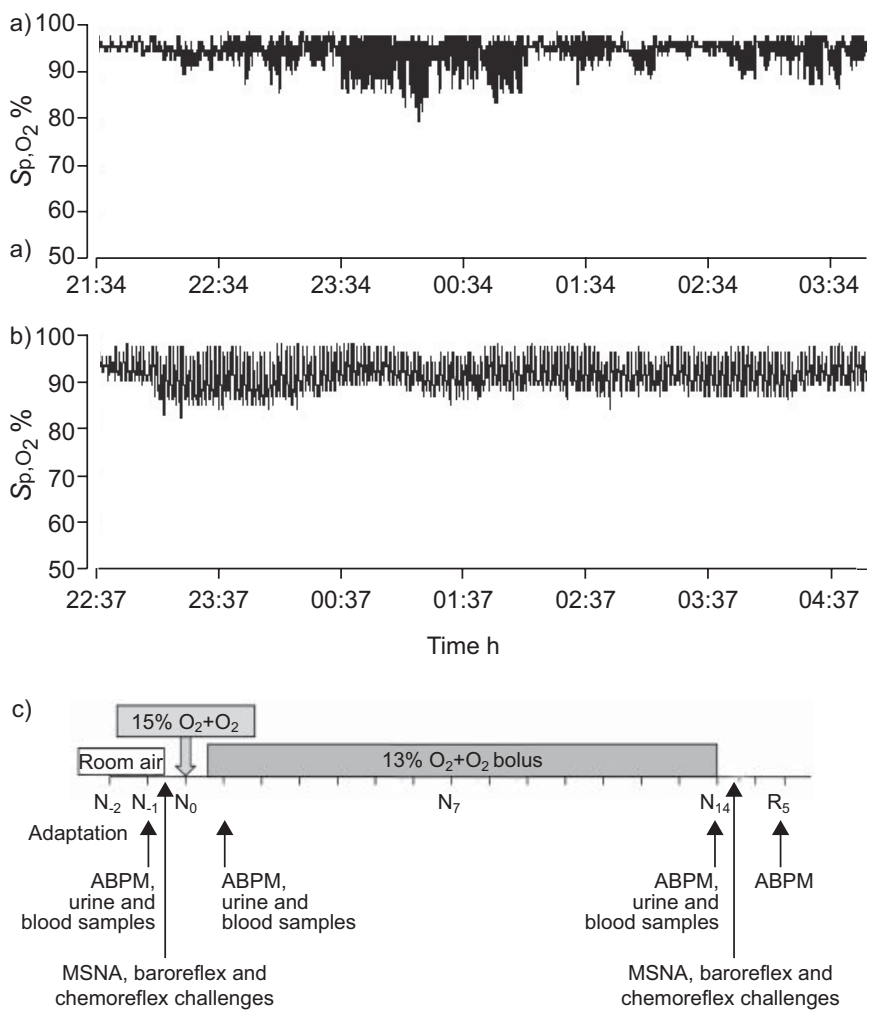

FIGURE 1. Arterial oxygen saturation measured by pulse oximetry $\left(\mathrm{Sp}, \mathrm{O}_{2}\right)$ tracings from a) a typical obstructive sleep apnoea patient compared to b) the exposure obtained with the present model in a representative subject. c) Time line of the study. Diurnal blood pressure patterns (ambulatory blood pressure measurement; ABPM) as well as urine and blood samples were obtained before exposure, after one and 13 nights of severe intermittent hypoxia $(\mathrm{IH})$, and after 5 days of recovery. Cardiovascular measurements (muscle sympathetic nerve activity; MSNA) and reflex assessments were performed before exposure and following the 2 weeks of severe $\mathrm{H}$. N: night; R: recovery.

as described previously [28]. Signals were filtered, amplified and full-wave rectified (Nerve Traffic Analyser, model 662c-3; Bioengineering Dept, University of Iowa, Iowa City, IA, USA), and muscle sympathetic nerve activity (MSNA) bursts were identified using an algorithm developed by HAMNER and TAYLOR [30], using Matlab software (The Mathworks Inc., Natick, MA, USA). MSNA was averaged over 5-min periods and expressed as burst frequency (bursts $\cdot \mathrm{min}^{-1}$ ), burst frequency normalised to heart beat (bursts per 100 beats) and burst amplitude $\left(\mathrm{AIU} \cdot \mathrm{min}^{-1}\right)$.

Calf blood flow (CBF) was measured by venous occlusion plethysmography (EC6 Plethysmograph; Hokanson, Bellevue, WA, USA), as described previously [28]. An average of eight to 10 flow measurements were used to compute values before and after exposure. CBF was expressed in millilitres per minute per $100 \mathrm{~g}$ tissue. Calf vascular resistance (CVR) was derived from the ratio of mean arterial pressure to CBF. These measures were also used to estimate the reactive hyperaemia over $2.5 \mathrm{~min}$ in response to $5 \mathrm{~min}$ local ischaemia.

Baroreflex control of sympathetic activity was assessed from concurrent beat-by-beat arterial pressures, MSNA and R-R intervals acquired during sequential bolus injections of $100 \mu \mathrm{g}$ nitroprusside followed $1 \mathrm{~min}$ later by $150 \mu \mathrm{g}$ phenylephrine (modified Oxford technique). Two trials were performed separated by $\geqslant 15 \mathrm{~min}$.

The sympathoexcitatory response to hypoxia was assessed from concurrent beat-by-beat arterial pressures, MSNA and R$\mathrm{R}$ intervals during a single hypoxic challenge. The hypoxic challenge was isocapnic to eliminate potential differences in end-tidal carbon dioxide across subjects. The target oxygen saturation for this test was $80-85 \%$. The mean \pm SD achieved was $80 \pm 3$ and $80 \pm 2 \%$ before and after exposure, respectively, and all subjects were between 78 and $85 \%$. Responses to hypoxia were measured during a 5 -min period only after steady state oxygen saturation was reached.

Transthoracic echocardiography (HP Sonos 2500; HewlettPackard, Santa Clara, CA, USA) was performed by the same investigator (J.P. Baguet, Dept of Cardiology and Hypertension, University Hospital, Grenoble, France) before and after exposure. Subjects were studied while in the left lateral decubitus position to obtain three standard left ventricular apical views (apical four-chamber, two-chamber and long-axis) using time motion, two-dimensional and Doppler modes with a $2.5-\mathrm{MHz}$ probe. Three stable and well-defined consecutive cardiac cycles were acquired for offline analysis of left ventricular dimensions, ejection fraction, stroke volume and ascending aorta diameter. This analysis was performed by the same investigator with an intra-observer reproducibility of $5 \%$.

\section{Data analysis}

When possible, data were acquired from 12 subjects. Ambulatory blood pressure measurements were not obtained on one subject after 5 days of recovery. The difficulty of obtaining and maintaining an adequate sympathetic neurogram resulted in incomplete data on four subjects either before or after exposure (one without pre-exposure, three without post-exposure recordings) and loss of data during the procedures in three more. Since the latter reduced the number of sympathetic recordings for the sympathoexcitatory response to hypoxia to only five subjects and, consequently, rendered paired sampling too small, data for these responses are presented only for discussion. In addition, for technical reasons, reactive hyperaemia was not obtained in one subject.

Ambulatory blood pressure monitoring over $24 \mathrm{~h}$ at 15 -min intervals results in a surfeit of values, such that comparisons of blood pressure measurements across the four study days on an hour-by-hour basis would be statistically intractable. Therefore, we averaged day- and night-time blood pressures. The classical definitions for day- (07:00-22:00 h) and night-time (22:00-07:00 h) measurements were applied to characterise diurnal blood pressure patterns.

Plasma markers for sympathetic activation (adrenaline, noradrenaline, dopamine, metanephrine and normetanephrine) were quantified by high-performance liquid chromatography. Nocturnal 8-h urine samples were collected, acidified with acetic acid and stored at $-20^{\circ} \mathrm{C}$ until analysis. Catecholamines (adrenaline, noradrenaline and dopamine) were measured in $1 \mathrm{~mL}$ of urine by high-performance liquid chromatography with electrochemical detection (Coularray Detector; ESA Dionex, Chelmsford, MA, USA). Plasma markers for systemic 
inflammatory responses (interleukin (IL)-1 receptor antagonist (IL-1Ra), IL-8, tumour necrosis factor (TNF)- $\alpha$, monocyte chemoattractant protein (MCP)-1, adiponectin, leptin and RANTES (regulated upon activation, normal T-cell expressed and secreted)) were quantified using a commercially available multiplex-bead immunoassay (R\&D Systems, Minneapolis, MN, USA) using a Bioplex 200 array reader (Bio-Rad, Hercules, CA, USA) with Luminex xMAP Technology (Luminex, Austin, TX, USA). Serum high-sensitivity C-reactive protein (hsCRP) level was measured using automated immunonephelometry (Behring Nephelometer II Analyser; Dade Behring, Berlin, Germany). Soluble intercellular adhesion molecule (sICAM)-1 was quantified by ELISA (British Biotechnology, Abingdon, UK).

Baroreflex function was estimated from the relation of systolic pressure to $\mathrm{R}-\mathrm{R}$ interval as described previously [31]. We assessed data from the pressure rise, since it represents baroreflex afferent activation of cardiac vagal outflow. Analysis began at the lowest pressure value after the bolus injection of nitroprusside and ended with the phenylephrineinduced peak. This selection of data points often encompasses threshold and saturation regions of the sigmoid relationship. To make the analysis objective and, therefore, independent of investigator bias, we analysed the data via a piecewise linear regression that required at least five data points to define the presence of threshold and/or saturation (if any). Arterial baroreflex control of MSNA was derived from the method developed by STUDINGER et al. [32]. Briefly, this technique excludes all data $2 \mathrm{mmHg}$ above the greatest pressure associated with a sympathetic burst and weights all cardiac cycles associated with zero sympathetic activity. Zeros above the highest pressure associated with a burst of activity are assigned a weight of 1 , and zeroes between the lowest and highest pressures are assigned a weight progressively increasing from 0 to 1 , proportional to the range of pressures observed. The linear gain for baroreflex-mediated sympathoinhibition is determined by eliminating threshold and/or saturation regions via piecewise linear regression. This approach provides a slope for baroreflex gain in an objective manner.

\section{Statistics}

Differences between the multiple means for ambulatory blood pressures and plasma markers were evaluated by ANOVA, corrected for multiple measures or a Friedman test when appropriate. When follow-up blood pressure data were missing, we assumed no change occurred to keep conclusions from the statistics conservative. When the ANOVA differences were detected $(\mathrm{p}<0.05)$, individual means were tested with the Bonferroni test. The Bonferroni correction for these repeated comparisons required p-values $<0.008$ to be considered statistically significant.

Comparison of cardiovascular measurements before exposure to those following 14 nights of severe $\mathrm{CIH}$ was via a paired, two-tailed t-test. For this, p-values $<0.05$ were considered statistically significant. Data are presented as mean \pm SD in the text, tables and figures.

\section{RESULTS}

Although $\mathrm{IH}$ was applied during sleep, increased ambulatory blood pressure occurred during the daytime and not the nighttime (fig. 2). The 24-h profile suggested that blood pressure increased prior to waking and then returned to normal, until increasing again over the late afternoon and into the evening. This daytime pattern became evident after only one night of exposure and was more sustained after 2 weeks of exposure. As a result, the average daytime blood pressure was increased for both mean and diastolic pressures after one and 13 nights of exposure $(3 \mathrm{mmHg}$ and $5 \mathrm{mmHg}$ for both; $\mathrm{p}<0.05$; fig. 3 ). At 2 weeks of exposure, there was a further significant increase in systolic blood pressure $(8 \mathrm{mmHg}$; $<<0.05$; fig. 3 ). Thus, the rise in blood pressure was sustained throughout the daytime, beyond the acute phase of severe $\mathrm{IH}$, but returned to baseline by 5 days of recovery.

Resting heart rate was unchanged following 2 weeks of $\mathrm{IH}$ exposure $\left(58.6 \pm 6.8\right.$ beats $\cdot \mathrm{min}^{-1}$ pre-exposure versus $59.4 \pm 7.6$ beats $\cdot \min ^{-1}$ post-exposure). After exposure, resting MSNA was elevated by $\sim 25 \%$ after the 14 day IH exposure ( $<<0.01$; fig. 4). Moreover, resting $\mathrm{CBF}$ was unchanged but there was a significant increase in CVR $\left(44.5 \pm 16.4 \mathrm{mmHg} \cdot \mathrm{min} \cdot \mathrm{mL}^{-1} \cdot 100\right.$ $\mathrm{mL}$ tissue pre-exposure versus $50.9 \pm 17.4 \mathrm{mmHg} \cdot \mathrm{min} \cdot \mathrm{mL}^{-1}$ $100 \mathrm{~mL}$ tissue post-exposure; $\mathrm{p}<0.05)$. In addition, the initial peak CBF following release of $5 \mathrm{~min}$ of ischaemia was lower following 2 weeks of $\mathrm{IH}\left(31.46 \pm 1.95 \mathrm{~mL} \cdot \mathrm{min}^{-1} \cdot 100 \mathrm{~g}\right.$ tissue $\mathrm{e}^{-1}$ pre-exposure versus $24.35 \pm 2.07 \mathrm{~mL} \cdot \mathrm{min}^{-1} \cdot 100 \mathrm{~g}$ tissue ${ }^{-1}$ postexposure; $\mathrm{p}<0.05$ ). However, from $30 \mathrm{~s}$ following the peak reactive hyperaemia, $\mathrm{CBF}$ values were similar before and after

\section{TABLE 1 Echocardiographic parameters monitored before and after exposure}

\begin{tabular}{|c|c|c|c|c|}
\hline Parameter & Subjects $\mathbf{n}$ & Pre-exposure & Post-exposure & p-value \\
\hline VTI cm & 12 & $20.7 \pm 2.2$ & $20.1 \pm 4.1$ & NS \\
\hline $\mathrm{CO} \mathrm{mL} \cdot \mathrm{min}^{-1}$ & 11 & $8374.1 \pm 1020.3$ & $7855.5 \pm 2040.9$ & NS \\
\hline LVEF \% & 10 & $67.8 \pm 2.7$ & $69.2 \pm 5.8$ & NS \\
\hline Heart rate beats $\cdot \mathrm{min}^{-1}$ & 11 & $66.9 \pm 9.7$ & $65.3 \pm 12.3$ & NS \\
\hline Stroke volume $\mathrm{mL}$ & 12 & $127.1 \pm 16.2$ & $124.6 \pm 30.1$ & NS \\
\hline Aortic diameter mm & 12 & $27.6 \pm 3.2$ & $28.4 \pm 2.9$ & 0.07 \\
\hline LV end-diastolic diameter $\mathrm{mm}$ & 12 & $50.2 \pm 4.5$ & $48.7 \pm 4.3$ & $<0.05$ \\
\hline LV end-systolic diameter $\mathrm{mm}$ & 12 & $33.8 \pm 3.2$ & $32.3 \pm 4.2$ & 0.06 \\
\hline
\end{tabular}

Data are presented as mean \pm SD, unless otherwise stated. VTI: velocity time integral ratio; CO: cardiac output; LVEF: left ventricular (LV) ejection fraction (Teicholz method); NS: not significant. 

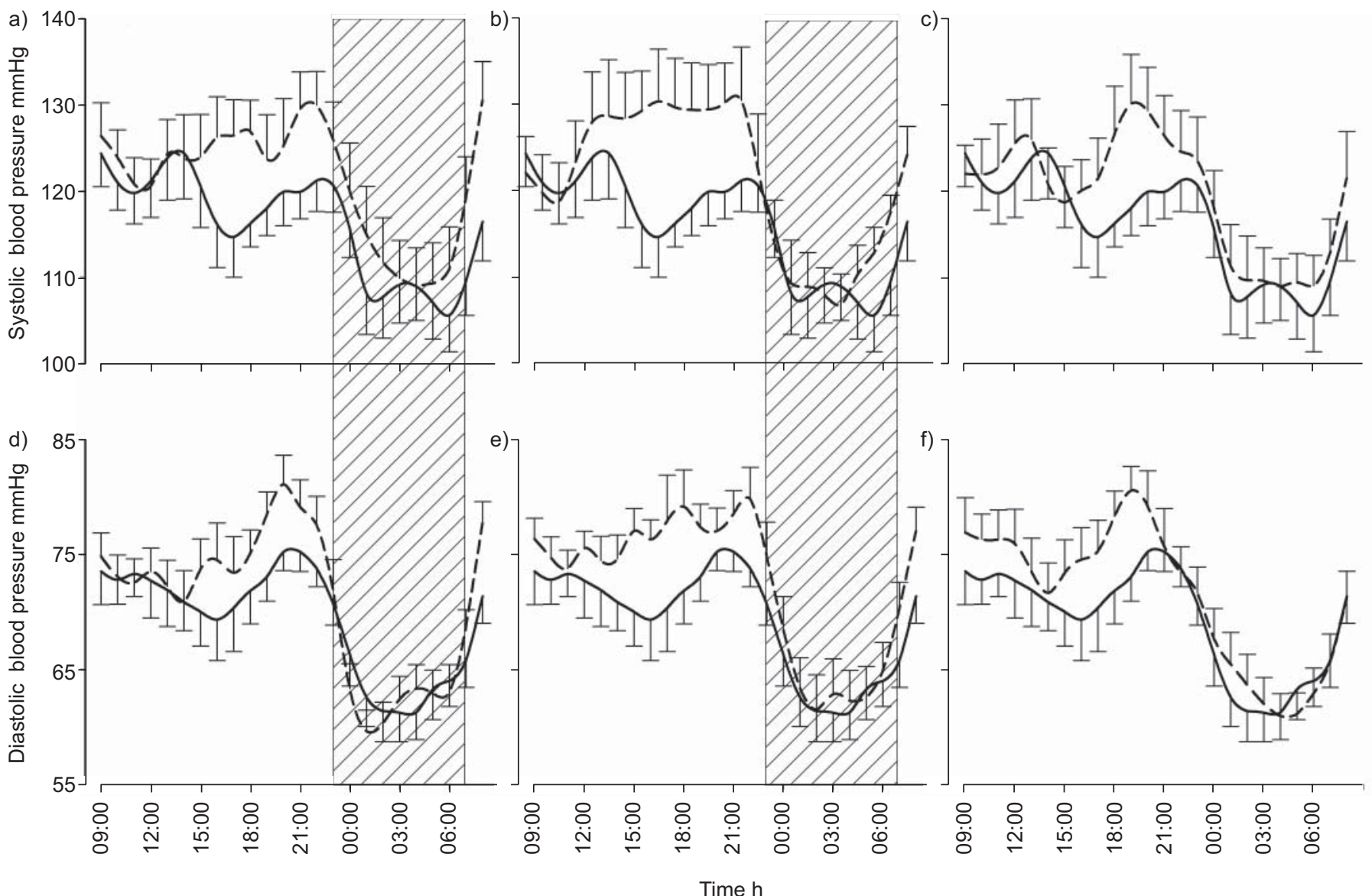

Time h

FIGURE 2. Hour-by-hour a, b, c) systolic and d, e, f) diastolic blood pressures during $24 \mathrm{~h}$ of monitoring ( $\mathrm{n}=12$ ). Data are presented as mean \pm SE. a, d) One night, b, e) 13 nights and c, f) recovery from exposure to intermittent hypoxia (Z) are compared to pre-exposure values. —-: pre-exposure; -----: post-exposure. Hourly values were averaged across the daytime and night-time for statistical analysis.

2 weeks of IH. Figure 5 shows a representative sequence of baroreflex testing, and the vagal and sympathetic baroreflex gains before and after exposure. 2 weeks of IH increased cardiovagal baroreflex gain from $21.7 \pm 8.0$ to $28.7 \pm 7.9 \mathrm{~ms}$. $\mathrm{mmHg}^{-1}(\mathrm{p}<0.05)$, but decreased vascular sympathetic gain from $-965.3 \pm 375.1$ to $-598.4 \pm 162.6 \mathrm{AIU} \cdot \mathrm{min}^{-1} \cdot \mathrm{mmHg}^{-1}$ $(\mathrm{p}<0.01)$.

Echocardiography showed that both left ventricular enddiastolic and -systolic diameters were reduced $(p<0.05$; table 1). As a result, ejection fraction and stroke volume were unchanged and, thus, cardiac output remained the same before and after exposure. Given the increase in daytime blood pressure, this indicates that systemic vascular resistance was elevated after 2 weeks of severe IH.

Despite the elevation in MSNA while awake, room air conditions, and urinary and plasma catecholamines demonstrated no change at any time during the severe $\mathrm{IH}$ exposure. Likewise, circulating plasma level of hsCRP, IL-1Ra, IL-8, TNF- $\alpha$, adiponectin, leptin, RANTES and sICAM- 1 did not change across the exposure, whereas MCP-1 tended to decrease $(p=0.06)$ across the exposure figure 6 .

To explore possible explanatory relationships for the increase in MSNA, we examined the correlation between the changes in
MSNA, left ventricular end-systolic and -diastolic diameters, and baroreflex sympathetic gain. The increase in MSNA with exposure was positively correlated with the decreases in both left ventricular end-systolic $(\mathrm{r}=0.73 ; \mathrm{p}<0.05)$ and -diastolic $(\mathrm{r}=0.72 ; \mathrm{p}=0.043)$ diameters. But, surprisingly, there was no relationship between the increase in MSNA and the decrease in baroreflex sympathetic gain $(r=0.46 ; p=0.294)$.

\section{DISCUSSION}

Our data clearly demonstrate that repeated exposure to an $\mathrm{IH}$ stimulus similar to that observed in severe OSAS patients produces a sustained daytime elevation in blood pressure in healthy humans. Moreover, this exposure increases resting sympathetic outflow and reduces sympathetic baroreflex gain. However, in our young healthy subjects, vasodilatory function was essentially unaltered and systemic markers of inflammation were virtually unchanged by severe $\mathrm{IH}$. However, it is possible that the time course for changes in these parameters requires a longer duration of exposure. Nonetheless, these data strongly suggest that exposure to severe $\mathrm{IH}$ in humans is responsible for a maintained elevation in blood pressure that is secondary, at least in part, to increased sympathetic activity and decreased baroreflex function.

Our human model of severe $\mathrm{IH}$ [25] produced particularly marked increases in pressure in the morning $(08: 00 \mathrm{~h})$ and 

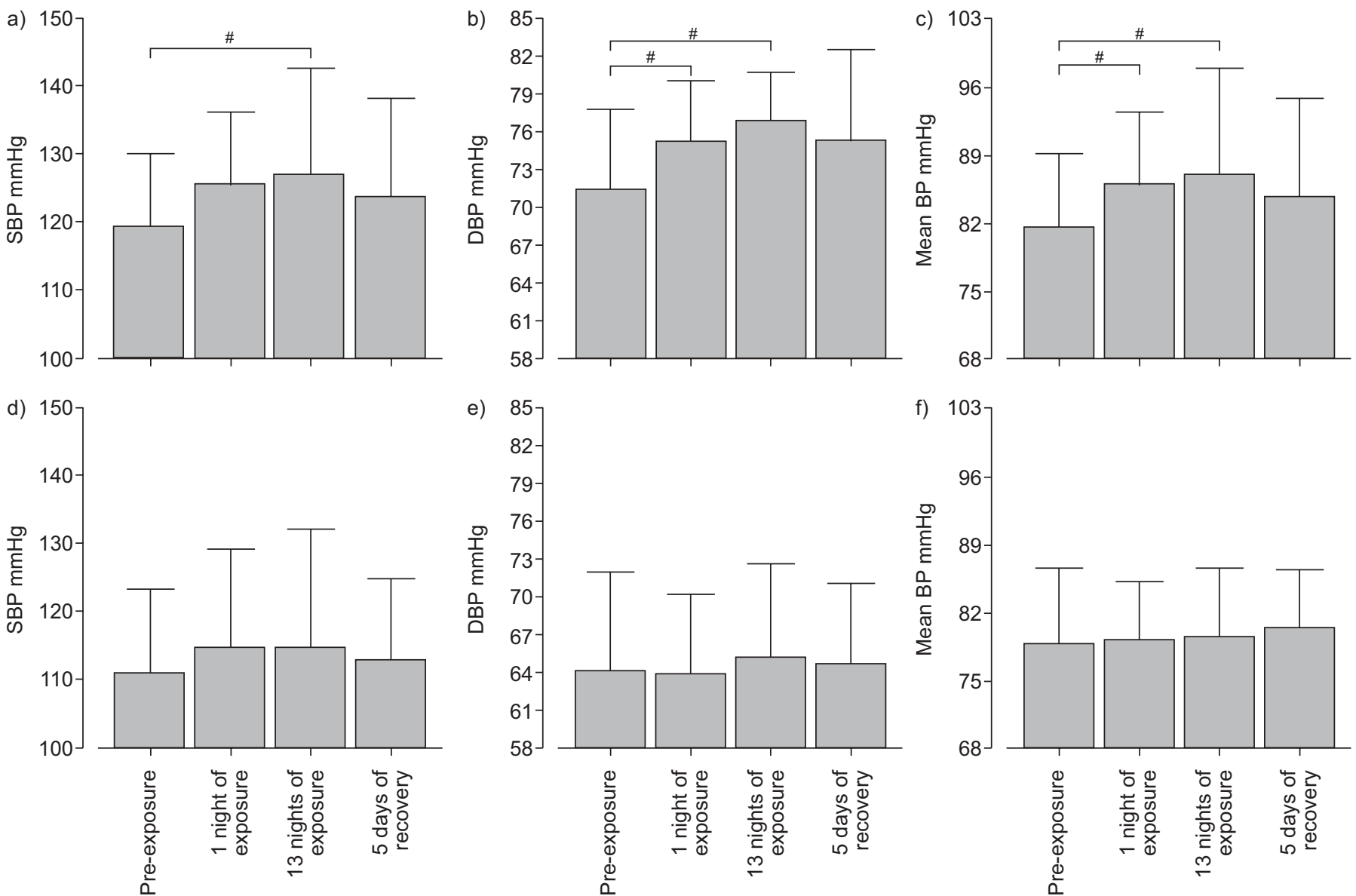

FIGURE 3. $a, b, c)$ daytime (07:00-22:00 h) and d, e, f) night-time (22:00-07:00 h) a, d) systolic blood pressure (SBP) b, e) diastolic blood pressure (DBP) and c, f) mean blood pressures (BP) across the exposure. Data are presented as mean \pm sD. $n=12 .{ }^{\#}: p<0.008$.

again later in the evening (18:00 and 20:00 h). In contrast, there was no night-time increase in blood pressure when subjects were exposed to IH during sleep. This resulted in an exaggeration of the normal nocturnal blood pressure decline, or "dip." This is in contrast to the classic "nondipper" profile that has been reported in $30 \%$ of OSAS patients [29]. We do not have definite explanation for this unexpected result. However, one point of note is that nocturnal catecholamine excretion did not change, and so it does not appear that there was any obvious alteration in sympathetic vasoconstrictor effects, and thus no effect on blood pressure. An alternative explanation is that nocturnal fall in blood pressure mainly relates to sleep duration and architecture. We recently demonstrated that, in type 1 diabetic OSA, shorter sleep duration, and not parameters of OSA severity, was the main determinant for non dipping pattern of blood pressue [33]. Our healthy subjects, even when hypoxaemic during the night, continued to exhibit a normal sleep organisation with a significant amount of slow wave sleep (time spent in stage III-IV sleep in $17.7 \pm 10.9 \%$ total sleep time (TST) before to $12.7 \pm 5.8 \%$ TST after exposure) [25]. This could lead to persistent physiological changes in the autonomic nervous system activity, resulting in a fall in blood pressure overnight. Interestingly, this was followed by persistent sympathetic hyperactivity during daytime, as evidenced by MSNA recordings.
Conversely, early morning and late afternoon increases in blood pressure have been described in OSAS patients [29], similar to what we observed after only 2 weeks of exposure to severe IH. In addition, it is important to note that the increased pressures induced by this protocol were resolved by 5 days of recovery. Though these data do not speak to the effect of longer exposures, the changes in blood pressure we observed suggest that increased daytime blood pressures can develop relatively quickly and that eliminating the stimulus for the sustained blood pressure elevation results in rapid resolution.

In tandem with this daytime elevation of pressure after 2 weeks of severe $\mathrm{IH}$, we observed a significant sympathetic activation. Although sympathetic activation with hypoxic exposure is thought to be mainly driven by increased peripheral chemoreflex sensitivity [8], we also observed a significant decrease in arterial baroreflex control of sympathetic outflow. However, there was not a simple linear relation between the increase in sympathetic activity and decrease in baroreflex gain. This does not exclude a baroreflex mechanism for the sympathoexcitation after $\mathrm{IH}$, but merely suggests the changes in baroreflex control do not necessarily lead to proportional changes in sympathetic activity. Lastly, we did find a greater cardiovagal gain after 2 weeks of $\mathrm{IH}$. More robust bradycardic responses to pressure rises might offset 
a)

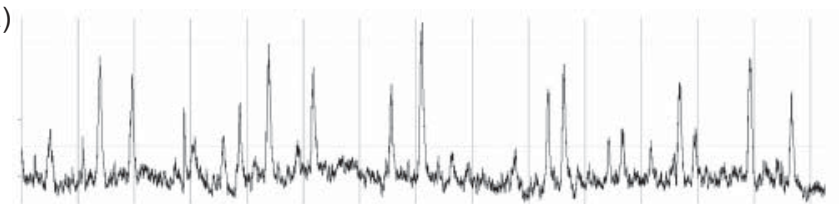

b)
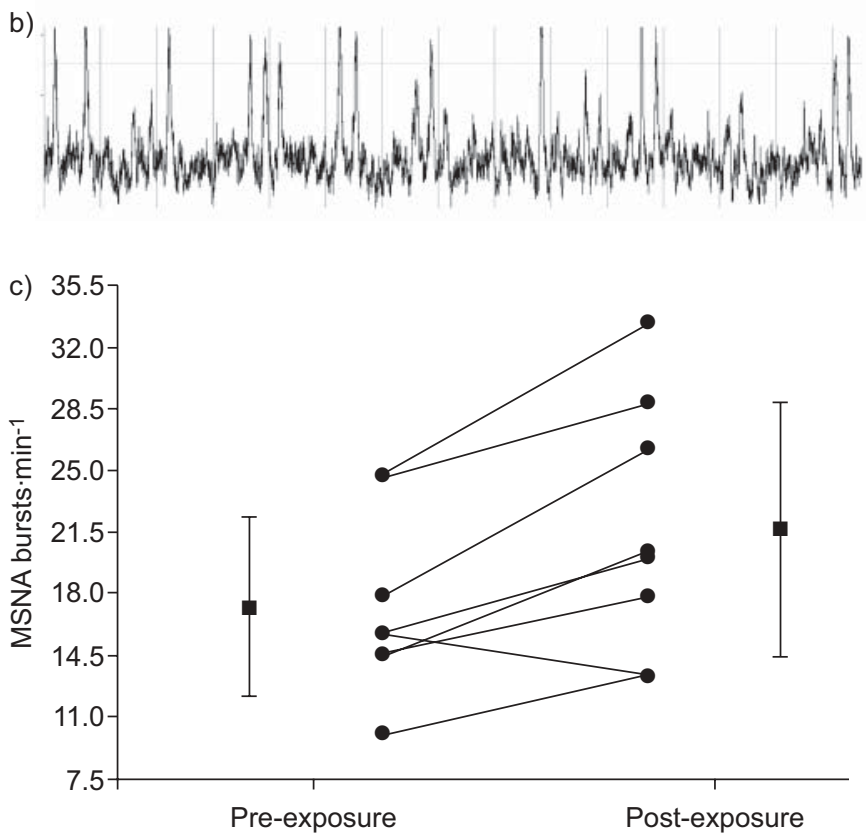

FIGURE 4. Representative neurograms of muscle sympathetic nerve activity (MSNA) during supine rest while breathing room air a) before and b) after 2 weeks intermittent hypoxia $(\mathrm{IH})$ exposure. c) The mean \pm SE values before and after 2 weeks $\mathrm{IH}$ exposure in bursts $\cdot \min ^{-1}(n=8 ; p=0.008)$.

lesser sympathoinhibition; however, these offsetting effects, if present, were apparently insufficient to prevent elevated ambulatory pressure after severe IH.

As noted above, peripheral chemoreflex sensitivity may also play a role in maintaining sympathoexcitation after exposure to hypoxia [8]. In a minority of subjects, we were able to obtain data suggestive of attenuation in the sympathoexcitatory responses to hypoxia. In our study, 14 nights of IH did tend to enhance the blood pressure increase in response to acute hypoxia $(11.0 \pm 6.9$ to $25.7 \pm 11.9 \mathrm{mmHg}$ for systolic and $2.3 \pm 3.0$ to $10.1 \pm 6.5 \mathrm{mmHg}$ for diastolic blood pressure before and after exposure, respectively). However, the magnitude of the sympathetic response in these five subjects with acute hypoxia appears to be diminished (pre-exposure $15.9 \pm 5.5$ to $27.0 \pm 11.9$ bursts $\cdot \mathrm{min}^{-1}$, post-exposure $21.0 \pm 7.5$ to $25.0 \pm 10.1$ bursts. $\left.\mathrm{min}^{-1}\right)$. This does not fit with the fact that prolonged exposure to hypoxia results in ventilatory acclimatisation to hypoxia: greater increases in ventilation [34] are due to augmented peripheral chemosensitivity [35]. Moreover, inhalation of $100 \% \mathrm{FI}, \mathrm{O}_{2}$ decreases sympathetic tone in OSAS patients [8], and surgical denervation of the peripheral chemoreceptors in rats prevents the increase in blood pressure induced by CIH [19]. Although these findings might suggest an increase in peripheral chemosensitivity as a mechanism of sympathoactivation, there is no standard approach to assess chemoreflex sensitivity in terms of sympathetic responsiveness. a) Sodium nitroprusside Phehylephrine
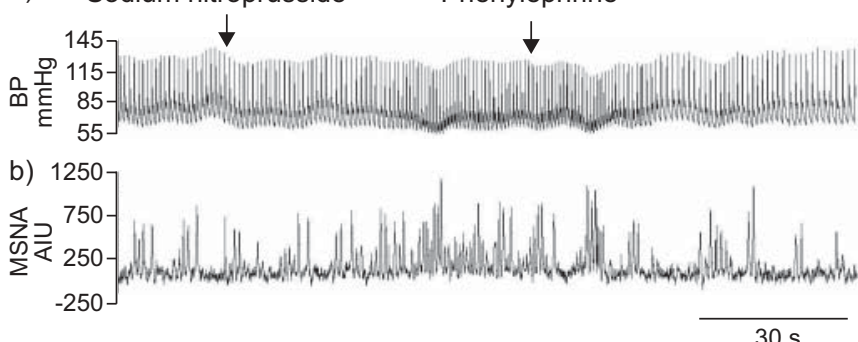

c) Sodium nitroprusside Phehylephrine
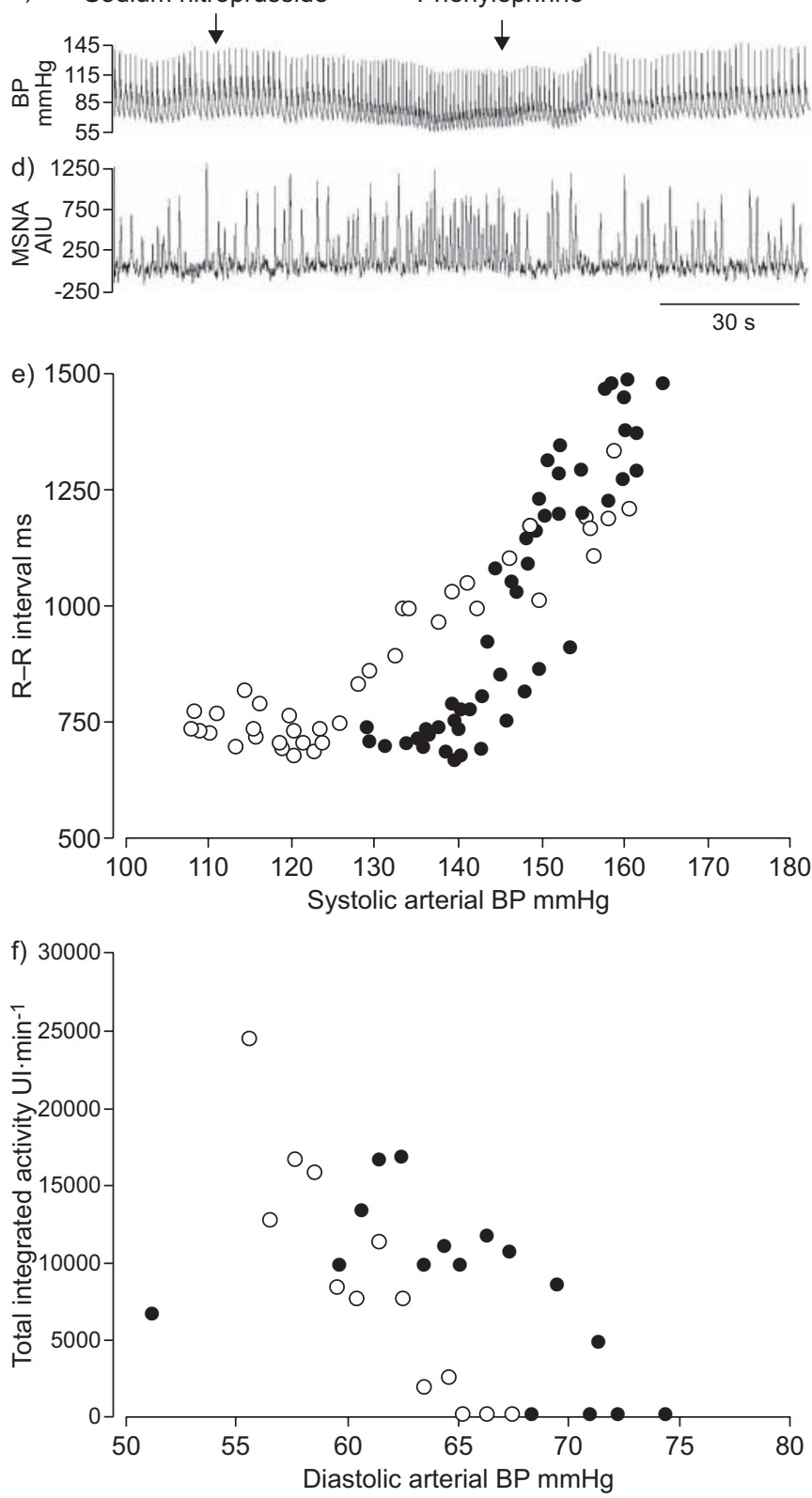

FIGURE 5. Representative sequences of arterial baroreflex testing a, b) before and $c, d$ ) after exposure and e, f) the relations derived from these time series. $\bigcirc$ before; - after. Analysis of these relations to obtain baroreflex gains is described in the text. BP: blood pressure; MSNA: muscle sympathetic nerve activity.

Hence, it remains unclear whether increased chemoreflex sensitivity plays a role in heightened sympathetic outflow after exposure to $\mathrm{CIH}$ in humans. 

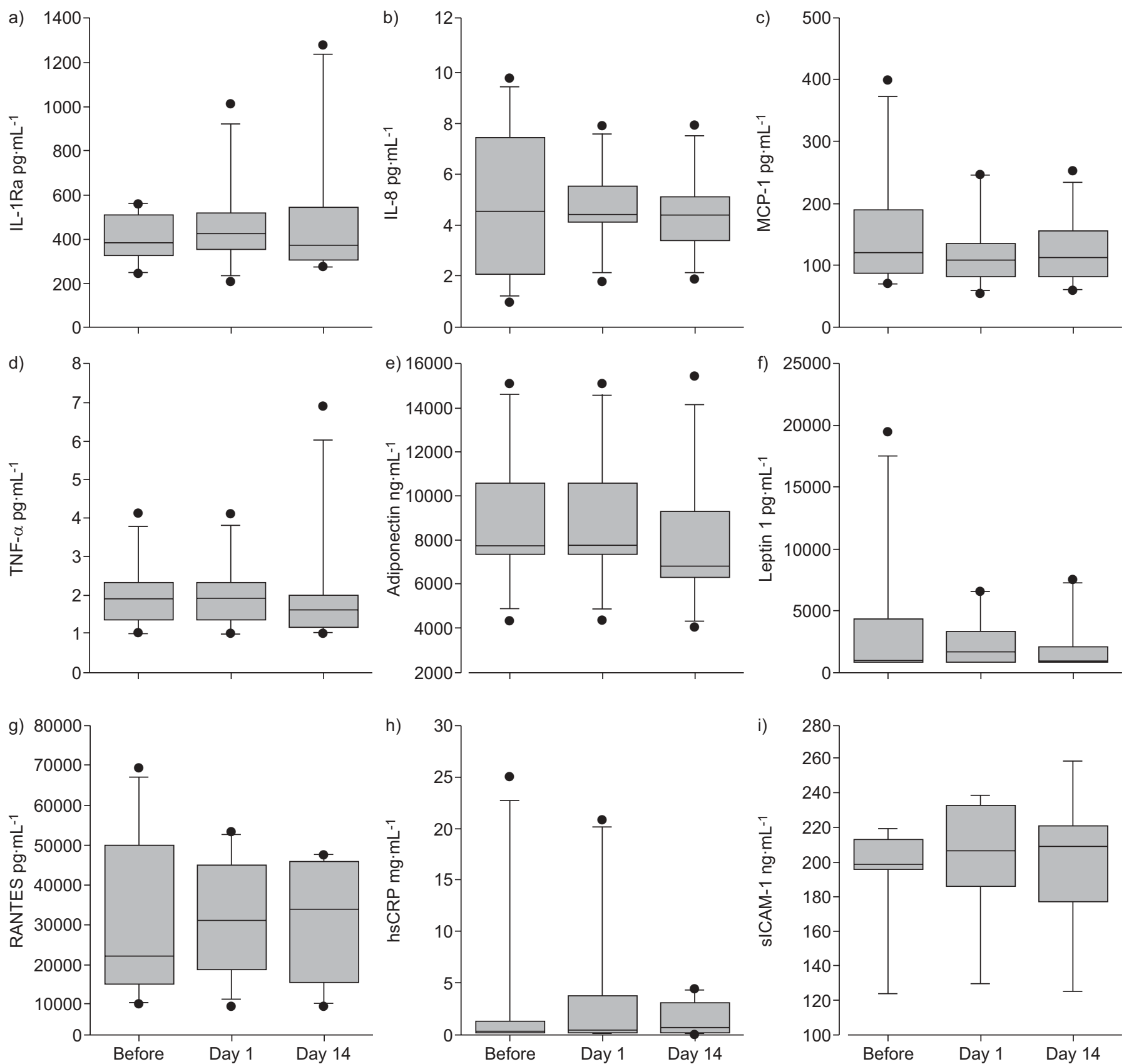

FIGURE 6. Box plots of circulating plasma levels of a) interleukin (IL)-1 receptor antagonist (Ra) $(p=0.91), b)$ IL-8 $(p=0.88), c)$ monocyte chemoattractant protein (MCP)-1 ( $p=0.06), d)$ tumour necrosis factor (TNF)- $\alpha(p=0.92), e)$ adiponectin $(p=0.29), f)$ leptin $(p=0.47), g)$ RANTES (regulated upon activation, normal T-cell expressed and secreted) $(p=0.89)$, h) high-sensitivity C-reactive protein (hSCRP) $(p=0.36)$ and i) soluble intercellular adhesion molecule ( $(\mathrm{ICAM})-1$ ( $p=0.44)$. Although MCP-1 tended to decrease during exposure, no significant change were found in other markers. The boxes represent interquartile range, and the whiskers represent the 5th and 95th percentiles. —: mean; - extremes. a) $p=0.91$, b) $p=0.88$, c) $p=0.06$, d) $p=0.92$, e) $p=0.29$, f) $p=0.47$, g) $p=0.89$, h) $p=0.36$ and i) $p=0.44$.

Several studies have proposed impaired vasodilation as a likely contributor to high blood pressure in OSAS patients [13, 36]. This work should be interpreted with caution, since many confounding factors (e.g. obesity and diabetes) may alter endothelial function independent of OSAS in these patients. In these young healthy subjects, we found a change only in the peak vasodilation following a hyperaemic stimulus. However, in contrast to previous studies in obstructive sleep apnoea patients [13, 37], we did not confirm an impairment in endothelially mediated vasodilation. We cannot rule out that a longer exposure to $\mathrm{CIH}$ might induce some change in vasodilatory capacity, but our data show that these changes are not necessary to observe the blood pressure increase with $\mathrm{CIH}$. Moreover, the systemic and endothelial inflammation reported in OSAS patients [15] and in rodent models of $\mathrm{CIH}$ [38] were not reproduced in our subjects.

Although given the number of desaturations (30 per hour), the stimulus we applied could be considered as clinically analogous to severe OSAS (fig. $1 \mathrm{a}$ and b); our model does 
have certain limitations and is not completely analogous to severe OSAS. Several points need to be discussed in order to present how our model differs from OSAS (fig. 1a and b). Although the amount of desaturation is close to a typical sleep apnoea patient, the timeline of $2 \mathrm{~min}$ per cycle is longer than that usually exhibited by a patient ( $<1 \mathrm{~min})$. Our exposure approximates IH paradigms applied to the rodent; however, it is closer to exposures in patients when breathing frequency is considered (about 16 breaths $\cdot \mathrm{min}^{-1}$ in humans versus 80 breaths. $\mathrm{min}^{-1}$ in rats). Moreover, apnoeas produce asphyxia (i.e. hypoxia plus hypercapnia), whereas our model produces hypoxia with hypocapnia. This may underestimate the effect, since increased carbon dioxide enhances the cardiovascular responses to hypoxia in both healthy individuals [39, 40] and sleep apnoea patients [41]. In addition, the absence of crescendo in respiratory effort will not result in the alterations in cardiac preload and afterload observed in sleep apnoea patients [42, 43]. However, the goal of this model is to explore a disease component to extricate a particular mechanism. Thus, the present model allows studying the specific effect of intermittent hypoxia during a specific physiological state: sleep. This stimulus, in this very young (23 yrs of age) and lean (BMI $22 \mathrm{~kg} \cdot \mathrm{m}^{-2}$ ) cohort, did not produce increased nocturnal catecholamine excretion, decreased daytime vagal drive or surges in nocturnal blood pressure. These responses have previously been well illustrated in obese, middle-aged, borderline hypertensive individuals with established sleep apnoea of $\geqslant 10$ yrs duration [42]. Therefore, it should be kept in mind that this model establishes that IH per se can lead to elevations in both blood pressure and vascular sympathetic activity over only a very short course of time. In addition, and as noted previously, the duration of exposure may not allow full resolution of other, potentially important pathophysiologic changes. 2 weeks of $\mathrm{IH}$ is likely a much shorter exposure than that experienced by individuals presenting with clinically significant OSAS. Finally, neither the renin-angiotensin system nor sodium balance were investigated in this study. Indeed, it would be interesting to explore several other mechanisms that may be involved in blood pressure increase, such as the renin-angiotensin system or endothelin pathway. Unfortunately, we did not include in our design sampling that would allows us to run a posteriori renin and angiotensin activity assays. This should be considered in the design of future studies.

\section{Future perspectives}

This is some of the first work to explore whether the blood pressure rise is sustained beyond the acute phase immediately after $\mathrm{IH}$ exposure, both throughout the waking hours and after 5 days of recovery. We found that only 2 weeks of severe $\mathrm{IH}$ exposure produces a sustained daytime blood pressure elevation in the setting of sympathoactivation and blunted vascular sympathic baroreflex gain in healthy individuals. This may provide a foundation from which interventional studies can be designed to explore prevention of the cardiovascular impairments due to $\mathrm{CIH}$.

\section{SUPPORT STATEMENT}

The study research was supported by grants from Direction de la Recherche Clinique (CHU Grenoble France), Agiradom and ANTADIR, and HL 74972 (J.W. Weiss and J.A. Taylor).

\section{STATEMENT OF INTEREST}

None declared.

\section{ACKNOWLEDGEMENTS}

We would like to thank P. Roux-Lombard (CHU Geneve, Geneva, Swizterland) who performed the multiplex and ELISA assays.

\section{REFERENCES}

1 Young T, Palta M, Dempsey J, et al. The occurrence of sleepdisordered breathing among middle-aged adults. N Engl J Med 1993; 328: 1230-1235.

2 Peppard PE, Young T, Palta M, et al. Prospective study of the association between sleep-disordered breathing and hypertension. N Engl J Med 2000; 342: 1378-1384.

3 Marin JM, Carrizo SJ, Vicente E, et al. Long-term cardiovascular outcomes in men with obstructive sleep apnoea-hypopnoea with or without treatment with continuous positive airway pressure: an observational study. Lancet 2005; 365: 1046-1053.

4 Gami AS, Howard DE, Olson EJ, et al. Day-night pattern of sudden death in obstructive sleep apnea. N Engl J Med 2005; 352: 1206-1214.

5 Yaggi HK, Concato J, Kernan WN, et al. Obstructive sleep apnea as a risk factor for stroke and death. $N$ Engl J Med 2005; 353: 2034-2041.

6 Levy P, Pepin JL, Arnaud C, et al. Intermittent hypoxia and sleepdisordered breathing: current concepts and perspectives. Eur Respir J 2008; 32: 1082-1095.

7 Bradley TD, Floras JS. Obstructive sleep apnoea and its cardiovascular consequences. Lancet 2009; 373: 82-93.

8 Narkiewicz K, van de Borne PJ, Montano N, et al. Contribution of tonic chemoreflex activation to sympathetic activity and blood pressure in patients with obstructive sleep apnea. Circulation 1998; 97: 943-945

9 Carlson JT, Hedner J, Elam M, et al. Augmented resting sympathetic activity in awake patients with obstructive sleep apnea. Chest 1993; 103: 1763-1768.

10 Hedner J, Darpo B, Ejnell H, et al. Reduction in sympathetic activity after long-term CPAP treatment in sleep apnoea: cardiovascular implications. Eur Respir J 1995; 8: 222-229.

11 Hedner J, Ejnell H, Sellgren J, et al. Is high and fluctuating muscle nerve sympathetic activity in the sleep apnoea syndrome of pathogenetic importance for the development of hypertension? J Hypertens Suppl 1988; 6: S529-S531.

12 Imadojemu VA, Gleeson K, Quraishi SA, et al. Impaired vasodilator responses in obstructive sleep apnea are improved with continuous positive airway pressure therapy. Am J Respir Crit Care Med 2002; 165: 950-953.

13 Ip MSM, Tse H-F, Lam B, et al. Endothelial function in obstructive sleep apnea and response to treatment. Am J Respir Crit Care Med 2004; 169: 348-353.

14 Kato M, Roberts-Thomson P, Phillips BG, et al. Impairment of endothelium-dependent vasodilation of resistance vessels in patients with obstructive sleep apnea. Circulation 2000; 102: 2607-2610.

15 Jelic S, Padeletti M, Kawut SM, et al. Inflammation, oxidative stress, and repair capacity of the vascular endothelium in obstructive sleep apnea. Circulation 2008; 117: 2270-2278.

16 Fletcher EC, Lesske J, Qian W, et al. Repetitive, episodic hypoxia causes diurnal elevation of blood pressure in rats. Hypertension 1992; 19: 555-561.

17 Brooks D, Horner RL, Kozar LF, et al. Obstructive sleep apnea as a cause of systemic hypertension. Evidence from a canine model. J Clin Invest 1997; 99: 106-109.

18 Fletcher EC, Lesske J, Culman J, et al. Sympathetic denervation blocks blood pressure elevation in episodic hypoxia. Hypertension 1992; 20: 612-619. 
19 Lesske J, Fletcher EC, Bao G, et al. Hypertension caused by chronic intermittent hypoxia: influence of chemoreceptors and sympathetic nervous system. J Hypertens 1997; 15: 1593-1603.

20 Greenberg HE, Sica A, Batson D, et al. Chronic intermittent hypoxia increases sympathetic responsiveness to hypoxia and hypercapnia. J Appl Physiol 1999; 86: 298-305.

21 Brooks D, Horner RL, Floras JS, et al. Baroreflex control of heart rate in a canine model of obstructive sleep apnea. Am J Respir Crit Care Med 1999; 159: 1293-1297.

22 Lai CJ, Yang CCH, Hsu YY, et al. Enhanced sympathetic outflow and decreased baroreflex sensitivity are associated with intermittent hypoxia-induced systemic hypertension in conscious rats. J Appl Physiol 2006; 100: 1974-1982.

23 Cook NR, Cohen J, Hebert PR, et al. Implications of small reductions in diastolic blood pressure for primary prevention. Arch Intern Med 1995; 155: 701-709.

24 Turnbull F. Effects of different blood-pressure-lowering regimens on major cardiovascular events: results of prospectively-designed overviews of randomised trials. Lancet 2003; 362: 1527-1535.

25 Tamisier R, Gilmartin GS, Launois SH, et al. A new model of chronic intermittent hypoxia in humans: effect on ventilation, sleep, and blood pressure. J Appl Physiol 2009; 107: 17-24.

26 Pialoux V, Hanly PJ, Foster GE, et al. Effects of exposure to intermittent hypoxia on oxidative stress and acute hypoxic ventilatory response in humans. Am J Respir Crit Care Med 2009; 180: 1002-1009.

27 Foster GE, Brugniaux JV, Pialoux V, et al. Cardiovascular and cerebrovascular responses to acute hypoxia following exposure to intermittent hypoxia in healthy humans. J Physiol 2009; 587: 3287-3299.

28 Tamisier R, Hunt BE, Gilmartin GS, et al. Hemodynamics and muscle sympathetic nerve activity after 8 hours of sustained hypoxia in healthy humans. Am J Physiol Heart Circ Physiol 2007; 293: H3027-3035.

29 Davies CW, Crosby JH, Mullins RL, et al. Case-control study of 24 hour ambulatory blood pressure in patients with obstructive sleep apnoea and normal matched control subjects. Thorax 2000; 55: 736-740.
30 Hamner JW, Taylor JA. Automated quantification of sympathetic beat-by-beat activity, independent of signal quality. J Appl Physiol 2001; 91: 1199-1206.

31 Studinger P, Goldstein R, Taylor JA. Mechanical and neural contributions to hysteresis in the cardiac vagal limb of the arterial baroreflex. J Physiol 2007; 583: 1041-1048.

32 Studinger P, Goldstein R, Taylor JA. Age- and fitness-related alterations in vascular sympathetic control. J Physiol 2009; 587: 2049-2057.

33 Borel AL, Benhamou PY, Baguet JP, et al. Short sleep duration is associated with a blood pressure nondipping pattern in type 1 diabetes: the DIAPASOM study. Diabetes Care 2009; 32: 1713-1715.

34 Powell FL, Milsom WK, Mitchell GS. Time domains of the hypoxic ventilatory response. Respir Physiol 1998; 112: 123-134.

35 Bisgard GE. Carotid body mechanisms in acclimatization to hypoxia. Respir Physiol 2000; 121: 237-246.

36 Kraiczi H, Hedner J, Peker Y, et al. Increased vasoconstrictor sensitivity in obstructive sleep apnea. J Appl Physiol 2000; 89: 493-498.

37 Jelic S, Lederer DJ, Adams T, et al. Vascular inflammation in obesity and sleep apnea. Circulation 2010; 121: 1014-1021.

38 Dematteis M, Julien C, Guillermet C, et al. Intermittent hypoxia induces early functional cardiovascular remodeling in mice. Am J Respir Crit Care Med 2008; 177: 227-235.

39 Morgan BJ, Crabtree DC, Palta M, et al. Combined hypoxia and hypercapnia evokes long-lasting sympathetic activation in humans. J Appl Physiol 1995; 79: 205-213.

40 Tamisier R, Nieto L, Anand A, et al. Sustained muscle sympathetic activity after hypercapnic but not hypocapnic hypoxia in normal humans. Respir Physiol Neurobiol 2004; 141: 145-155.

41 Kara T, Narkiewicz K, Somers VK. Chemoreflexes: physiology and clinical implications. Acta Physiol Scand 2003; 177: 377-384.

42 Somers VK, Dyken ME, Clary MP, et al. Sympathetic neural mechanisms in obstructive sleep apnea. J Clin Invest 1995; 96: 1897-1904.

43 Katragadda S, Xie A, Puleo D, et al. Neural mechanism of the pressor response to obstructive and nonobstructive apnea. J Appl Physiol 1997; 83: 2048-2054. 